\title{
Bistatic TerraSAR-X/F-SAR Spaceborne-Airborne SAR Experiment: Description, Data Processing, and Results
}

\author{
Marc Rodriguez-Cassola, Stefan V. Baumgartner, \\ Gerhard Krieger, Member, IEEE, and Alberto Moreira, Fellow, IEEE
}

\begin{abstract}
We report about the first X-band spaceborneairborne bistatic synthetic aperture radar (SAR) experiment, conducted early November 2007, using the German satellite TerraSAR-X as transmitter and the German Aerospace Center's (DLR) new airborne radar system F-SAR as receiver. The importance of the experiment resides in both its pioneering character and its potential to serve as a test bed for the validation of nonstationary bistatic acquisitions, novel calibration and synchronization algorithms, and advanced imaging techniques. Due to the independent operation of the transmitter and receiver, an accurate synchronization procedure was needed during processing to make high-resolution imaging feasible. Precise phase-preserving bistatic focusing can only be achieved if time and phase synchronization exist. The synchronization approach, based on the evaluation of the range histories of several reference targets, was verified through a separate analysis of the range and Doppler contributions. After successful synchronization, nonstationary focusing was performed using a bistatic backprojection algorithm. During the campaign, stand-alone TerraSAR-X monostatic as well as interoperated TerraSAR-X/F-SAR bistatic data sets were recorded. As expected, the bistatic image shows a space-variant behavior in spatial resolution and in signal-to-noise ratio. Due to the selected configuration, the bistatic image outperforms its monostatic counterpart in almost the complete imaged scene. A detailed comparison between monostatic and bistatic images is given, illustrating the complementarity of both measurements in terms of backscatter and Doppler information. The results are of fundamental importance for the development of future nonsynchronized bistatic SAR systems.
\end{abstract}

Index Terms-Bistatic radar, bistatic SAR processing, synchronization algorithms, synthetic aperture $\operatorname{radar}(\mathrm{SAR})$.

\section{INTRODUCTION}

$\mathbf{T}$ HE FIRST published results of a bistatic synthetic aperture radar (SAR) experiment date back to the mid-1980s [1]. The innovative bistatic radar consisted of two X-band airborne systems flying in parallel trajectories with an azimuthinvariant configuration. This experiment sparked the interest in inherent bistatic SAR issues like basic clock and antennafootprint synchronization, performance prediction, or focus-

Manuscript received September 22, 2008; revised March 16, 2009 and June 15, 2009. First published October 30, 2009; current version published January 20, 2010.

The authors are with the Microwaves and Radar Institute, German Aerospace Center (DLR), 82234 Oberpfaffenhofen, Germany (e-mail: marc.rodriguez@ dlr.de; stefan.baumgartner@dlr.de; gerhard.krieger@dlr.de; alberto.moreira@ dlr.de).

Color versions of one or more of the figures in this paper are available online at http://ieeexplore.ieee.org.

Digital Object Identifier 10.1109/TGRS.2009.2029984 ing, arising as new and challenging research topics. The next relevant experiments, packed in a series of $\mathrm{C}$ - and $\mathrm{L}$-band acquisitions involving ERS-1 and SIR-C as transmitters and a radar mounted on a NASA DC-8 as receiver, did not take place until the late 1990s and demonstrated the feasibility of a more complex acquisition scenario [2]. Valid bistatic data recording was ensured by detecting the energy of the direct signal received from the satellite. The innovative nature of the experiments described in [1] and [2] is rather obvious. However, the published results revealed a greater effort in the development of the campaign plannings than in the full analysis of the acquired bistatic data, and only low-resolution quick-look images of both experiments were published [2], [3]. From late 2002 onward, several bistatic X-band airborne SAR experiments were conducted in Europe showing the capabilities of bistatic SAR as a high-resolution imaging system [4]-[6]. In particular, the DLR-ONERA bistatic experiment pushed further the bistatic processing standard and stands as the first (and only, so far) demonstration of airborne cross-platform bistatic SAR interferometry [7]-[10]. Interferometric applications, for which accurate focusing is essential, constitute the basis of future operational spaceborne bistatic SAR missions like TanDEM-X [11]. The interest on bistatic SAR has rapidly increased in the last years, and several bistatic experiments with very different sensors and configurations have been conducted lately [12]-[15].

The advantages and disadvantages of bistatic SAR are a consequence of the spatial separation between transmitter and receiver. The disadvantages are mainly of technical nature and related to the increased complexity of system operation and data processing. The advantages include higher operational versatility, system design flexibility, and increased performance. In the case of hybrid spaceborne-airborne observations, the bistatic SAR system is more robust to jamming than both the spaceborne and the airborne systems operated monostatically. In addition, it delivers, for equivalent synthetic apertures, a better performance in terms of signal-to-noise ratio (SNR) and spatial resolution with respect to the monostatic spaceborne SAR. This paper presents the design, analysis, processing, and results of the first X-band spaceborne-airborne bistatic SAR experiment using TerraSAR-X as transmitter and the DLR's new airborne radar F-SAR as receiver [16], [17]. The experiment was proposed by the authors to the TerraSAR-X scientific coordination board in 2005 and was successfully conducted early November 2007. The flexibility of both radar systems, 
operated in nonnominal modes, turned out to be an essential advantage in the fast preparation of the campaign, ensuring the recording of bistatic data without significant hardware modifications. The experiment was carried out during the TerraSAR-X commissioning phase, and it served as a valuable input for testing the suitability of TerraSAR-X to produce high-quality bistatic images. The investigations made during campaign planning and data evaluation were essential in the validation of the whole processing chain for nonsynchronized short-time bistatic acquisitions, including calibration, synchronization, and highresolution focusing techniques.

The technical content of this paper is divided into four parts. Section II describes the bistatic configuration and acquisition. Section III presents the performance analysis of the bistatic system, showing the expected values of ground-range and along-track resolutions, as well as predictions of the noiseequivalent sigma zero (NESZ). The influence of the nonstationary antenna pattern modulation on the resolution and the SNR is discussed and quantified. Section IV describes the processing of the acquired data. Given the acquisition scenario, data processing must undergo a three-step procedure: 1) channel balancing; 2) data synchronization; and 3) azimuthvariant bistatic SAR focusing. The bistatic image is presented and analyzed in Section V. The evaluation includes a point response and residual phase error analysis. A comparison between monostatic and bistatic images is also presented at the end of this section. Section VI summarizes the results.

\section{DESCRIPTION OF THE EXPERIMENT}

\section{A. Bistatic Configuration}

The trajectory of F-SAR was designed to be nearly parallel to the nadir track of TerraSAR-X to maximize footprint overlapping time. Bistatic side-looking operation was preferred to guarantee a good ground-range resolution. F-SAR was operated as a receiver and flew at a height of $2180 \mathrm{~m}$ above ground with a mean velocity of $90 \mathrm{~m} / \mathrm{s}$. TerraSAR-X transmitted on a 514-km orbit with a velocity of $7600 \mathrm{~m} / \mathrm{s}$. The TerraSAR-X antenna was steered in high-resolution (sliding) spotlight mode. Two crucial details distinguished the acquisition from a nominal TerraSAR-X high-resolution spotlight: 1) The transmitted bandwidth was reduced from 300 to $100 \mathrm{MHz}$, and 2) the pulse repetition frequency (PRF) was optimized only for the bistatic acquisition. Fig. 1 shows the geometry of the bistatic TerraSAR-X/F-SAR acquisition, with $51^{\circ}$ and $45^{\circ}$ being the off-nadir angles of transmitter and receiver, respectively. The onground depicted footprint coincides with the instantaneous one-way 3-dB footprint of F-SAR. The previous off-nadir angles correspond to nominal midbeam incident angles of $55.63^{\circ}$ for TerraSAR-X and $45^{\circ}$ for F-SAR. Due to the effect of the curved path of the satellite, the equivalent velocity in monostatic midrange was $7408 \mathrm{~m} / \mathrm{s}$. The bistatic experiment was performed in Germany near the Kaufbeuren airfield, which is used as a calibration test site by the DLR Microwaves and Radar Institute. In order to avoid conflicts with other acquisitions and tests performed over Kaufbeuren during the TerraSAR-X commissioning phase, the satellite was exceptionally operated in left-looking mode.

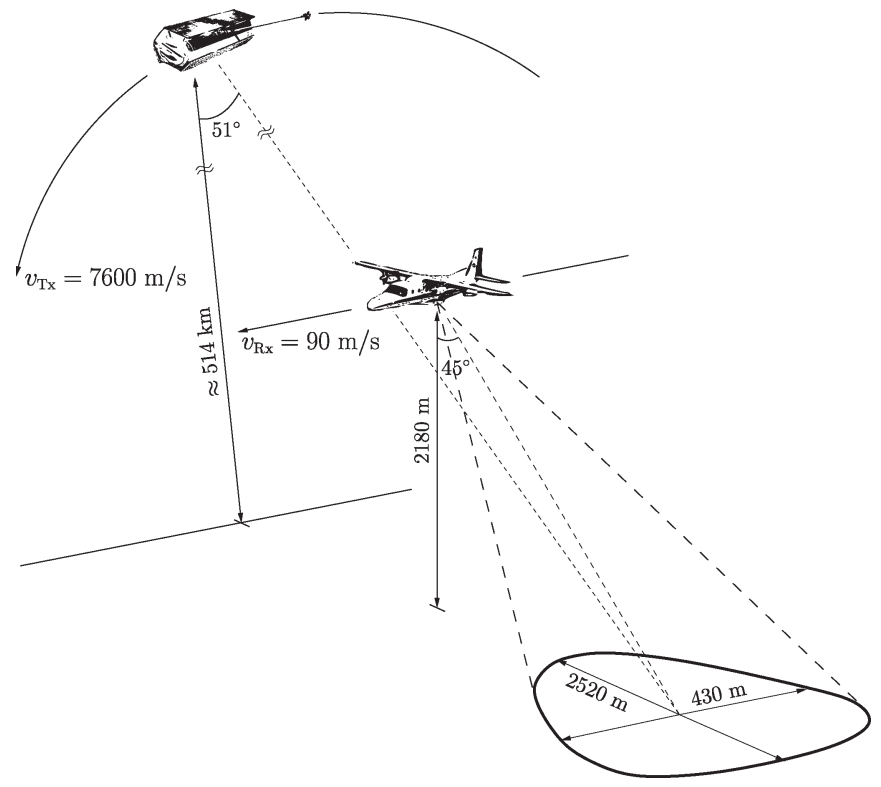

Fig. 1. Bistatic configuration in the TerraSAR-X/F-SAR spaceborne-airborne SAR experiment.

\section{B. Bistatic Acquisition}

The quasi-continuous data-acquisition capability of F-SAR is a prominent advantage of using it as a receiver [18]. This feature simplified the campaign preparation and avoided the need for sophisticated echo-window synchronization. Due to data-rate constraints, two analog-to-digital converters (ADCs) (cf. Fig. 2) had to be switched alternately to enable quasi-continuous data recording [16], [17]. To avoid any hardware modification on the F-SAR X-band receiver chain, not only two different ADCs but also two different receiver chains were used. Switching between both channels produced a sampling blank interval of $1.6 \mu$ s and was performed with a $7693.25-\mathrm{Hz}$ cadence. The maximum real sampling rate using this configuration was $250 \mathrm{MHz}$, and the selected transmitted chirp bandwidth was $100 \mathrm{MHz}$. The down-converting reference frequency in the receiver was matched to the nominal value of the TerraSAR-X carrier $(9.65 \mathrm{GHz})$. Chirp duration was $33.189 \mu \mathrm{s}$, and the transmitted peak power was $2.01 \mathrm{~kW}$. As stated in the previous section, the TerraSAR-X antenna beam was steered in azimuth to perform a high-resolution spotlight illumination of the scene so that footprint overlapping time was maximized. The F-SAR received in regular stripmap mode. A high transmit PRF of $5920.59 \mathrm{~Hz}$ was chosen to guarantee a high along-track oversampling rate of the bistatic data. Since the joint antenna footprint is limited by the F-SAR antenna pattern, no range ambiguities are expected in the bistatic image. However, range ambiguities arise in the monostatic image, adversely affected by the atypically shallow look angle of the satellite. We would like to stress that the range ambiguities expected in the monostatic image are a consequence of the non-monostatic-optimized setup defined for the acquisition. In a nominal TerraSAR-X monostatic high-resolution spotlight image, the expected range ambiguity-to-signal ratio will be significantly better than the one obtained in the monostatic image of Section V.

Fig. 2 shows the block diagram of the bistatic radar used for the spaceborne-airborne acquisition. The first main 
TSX/Tx

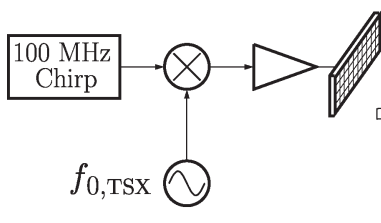

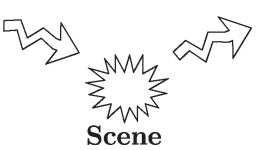

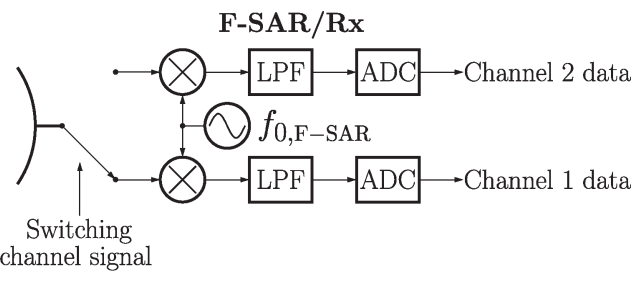

Fig. 2. Bistatic radar block diagram of the spaceborne-airborne TerraSAR-X/F-SAR experiment. The left part shows the transmitter (TerraSAR-X), and the right part shows the receiver (F-SAR) with its two different channels.

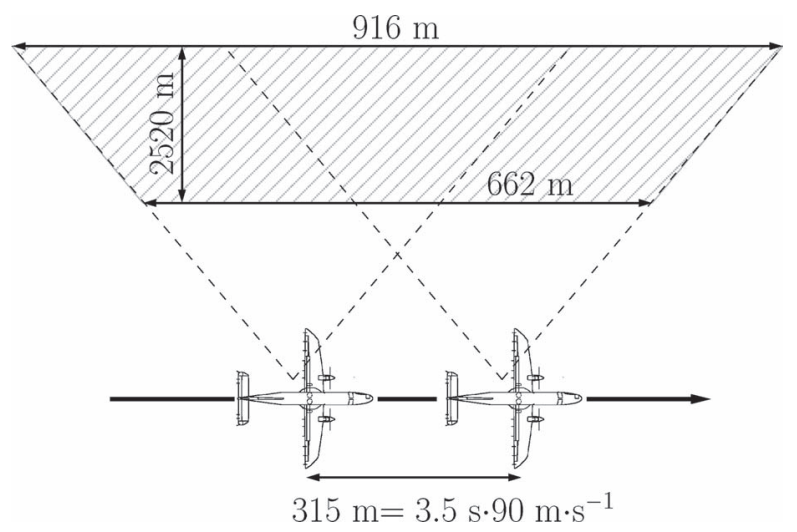

Fig. 3. Bistatic scene dimensions in the TerraSAR-X/F-SAR bistatic experiment. Dimensions are defined by the motion of the F-SAR 3-dB one-way antenna footprint during the bistatic acquisition.

difference with respect to a monostatic configuration is the use of two different clocks working independently, $f_{0, T S X}$ and $f_{0, \mathrm{~F}-\mathrm{SAR}}$, where the subscript TSX stands for TerraSAR-X. The left part of the plot shows the TerraSAR-X transmitter with its active antenna, and the right part shows the two receiving channels of F-SAR. Taking account of the differences in transmitted power, antennas, and free-space losses of this bistatic acquisition compared with a pure airborne monostatic case, the F-SAR receiving gain was increased by about $22 \mathrm{~dB}$ with respect to the monostatic operation mode. Three X-band transponders already used for the TerraSAR-X calibration were placed as reference targets. Their exact positions were measured with a GPS. These reference targets were essential not only to quantify the quality of the focused image, but also to precisely synchronize the data, as explained in Section IV.

\section{Imaged Scene}

With respect to F-SAR $(0.2 \mathrm{~m}$ in azimuth and $0.046 \mathrm{~m}$ in elevation), the TerraSAR-X antenna size (4.784 $\mathrm{m}$ in azimuth and $0.7 \mathrm{~m}$ in elevation) is larger, but its footprint on the ground is wider due to longer slant ranges. The bistatic footprint thus coincided with the F-SAR footprint during the time TerraSAR-X illuminated the scene. The footprint overlapping time was limited by the standard duration of TerraSAR-X high-resolution spotlight acquisitions. Bistatic data were recorded for approximately $3.5 \mathrm{~s}$. Within the $3-\mathrm{dB}$ region of the one-way F-SAR antenna pattern, the ground-range dimension of the imaged scene was $2520 \mathrm{~m}$. During the $3.5 \mathrm{~s}$ of the acquisition, the along-track size of the scene varied between 662 and $916 \mathrm{~m}$ for near and far ranges, respectively. Fig. 3 shows how the 3-dB instantaneous footprint of F-SAR moved during the ac-
TABLE I

Simulation Parameters for Performance ANALysis

\begin{tabular}{lcc}
\hline Integration time [s] & $T_{\mathrm{int}}$ & 2.77 \\
Scene dimensions [m $\times \mathbf{m}]$ & $L_{r} \times L_{a}$ & $7680 \times 2560$ \\
Wave propagation speed [m/s] & $\mathrm{c}$ & $2.9979 \cdot 10^{8}$ \\
Boltzmann constant [J/K] & $\mathrm{k}$ & $1.38 \cdot 10^{-23}$ \\
Wavelength [m] & $\lambda$ & 0.031 \\
Transmitted bandwidth [MHz] & $B_{r}$ & 100 \\
Average transmitted power [W] & $P_{\mathrm{Tx}}$ & 395 \\
Antenna gain Tx [dBi] & $G_{\mathrm{Tx}}$ & 46.41 \\
Antenna gain Rx [dBi] & $G_{\mathrm{Rx}}$ & 20.80 \\
TerraSAR-X effective velocity [m/s] & $v_{\mathrm{Tx}}$ & 7408 \\
F-SAR velocity [m/s] & $v_{\mathrm{Rx}}$ & 90 \\
TerraSAR-X altitude [km] & $h_{\mathrm{Tx}}$ & 514 \\
F-SAR altitude [m] & $h_{\mathrm{Rx}}$ & 2180 \\
Noise figure + losses [dB] & $F_{\mathrm{Rx}}$ & 4.5 \\
Rx system temperature [K] & $T_{n}$ & 300 \\
\hline
\end{tabular}

quisition, resulting in the expected dimensions of the bistatic scene. However, since the bistatic range was shorter than the satellite monostatic range, the attenuation due to the receive antenna pattern was partially compensated by the better SNR values of the bistatic image. Thus, a larger processed scene can be considered. The dimensions of the scene used for the performance analysis are $7680 \mathrm{~m}$ in ground range and $2560 \mathrm{~m}$ in azimuth. They coincide with those of the bistatic image shown in Section V. For targets placed at the along-track edges of the image, a significant portion of the integration is performed well outside the 3-dB range of the receive antenna. This effect has a direct impact on the along-track resolution and SNR of the bistatic image as discussed in Section III.

\section{Performance Analysis}

Due to the variance of this bistatic SAR acquisition, conventional performance analysis no longer holds. For the computation of the expected performance, a simplified form of the bistatic configuration is assumed, with a spherical Earth model and both platforms reaching zero-azimuth time at the same instant. The midrange incident angles are $55^{\circ}$ and $45^{\circ}$ for transmitter and receiver, respectively, and both antennas are pointing exactly at the same scene center. The parameters used in the simulations are presented in Table I. Furthermore, spotlight processing of the whole scene is considered. The scene dimensions are $7680 \mathrm{~m}$ in ground range and $2560 \mathrm{~m}$ in azimuth. The coordinates origin coincides with the $45^{\circ}$ incident angle of F-SAR. In addition to the particularities of bistatic imaging, the nonnegligible amplitude modulation introduced by the bistatic azimuth antenna pattern has to be taken into account in the derivation of azimuth resolution and SNR. 


\section{A. SNR and NESZ}

The expression in [19] is a valid approximation for the NESZ at the center of the image, where the joint antenna pattern is substituted by a constant gain factor $G_{\mathrm{Tx}} \cdot G_{\mathrm{Rx}}$ over the whole aperture. The NESZ of the bistatic system is approximated as

$$
\mathrm{NESZ} \approx \frac{(4 \pi)^{3} \cdot r_{0, \mathrm{Tx}}^{2} \cdot r_{0, \mathrm{Rx}}^{2} \cdot \mathrm{k} \cdot T_{n} \cdot F_{\mathrm{Rx}}}{P_{\mathrm{Tx}} \cdot G_{\mathrm{Tx}} \cdot G_{\mathrm{Rx}} \cdot \lambda^{2} \cdot A_{\text {cell }} \cdot T_{\mathrm{int}}}
$$

where $A_{\text {cell }}$ is the area of the resolution cell of the bistatic image. The NESZ takes on values around $-43 \mathrm{~dB}$ at the center of the scene, which is more than $20 \mathrm{~dB}$ better than the monostatic TerraSAR-X requirement. This increase in SNR is mainly due to the much shorter range from the bistatic receiver to the imaged area. The bistatic image is thus expected to have a far better sensitivity than its monostatic counterpart. However, the amplitude modulation introduced by the antenna patterns cannot be neglected in SNR and NESZ computations. Consequently, a key question to address is how the antenna pattern modulation modifies the signal and noise power distributions over the SAR image. In other words, it has to be established, for any given pixel, how much energy is due to the signal and how much is due to noise.

In our case, noise power presents a spatially uniform distribution in raw and range-compressed data. Due to the spotlight processing, the same number of samples is used in the computation of any image pixel. Since noise and signal are uncorrelated, SAR processing does not change the noise-power distribution in the SAR image. Assuming (1), the pixel noise power density after SAR focusing can be expressed as

$$
N \approx \frac{\mathrm{k} \cdot \mathrm{T}_{\mathrm{n}} \cdot \mathrm{F}_{\mathrm{Rx}}}{\mathrm{A}_{\mathrm{cell}} \cdot \mathrm{T}_{\mathrm{int}}} .
$$

Using similar approximations to those leading to (1), the signal power for a target area centered at coordinates $\left(r_{0, \mathrm{Tx}}, r_{0, \mathrm{Rx}}, x_{0}\right)$ can be expressed as

$$
\begin{aligned}
S\left(x_{0}\right) \approx & \frac{P_{\mathrm{Tx}} \cdot G_{\mathrm{Tx}} \cdot G_{\mathrm{Rx}} \cdot \lambda^{2}}{(4 \pi)^{3} \cdot r_{0, \mathrm{Tx}}^{2} \cdot r_{0, \mathrm{Rx}}^{2}} \\
& \cdot\left|\frac{1}{T_{\mathrm{int}}} \cdot \int_{T_{\mathrm{int}}} \sqrt{\sigma\left(x_{0}\right)} \cdot w_{\mathrm{Rx}}\left(t_{a} ; r_{0, \mathrm{Rx}}, x_{0}\right) \cdot d t_{a}\right|^{2}
\end{aligned}
$$

where $\sigma\left(x_{0}\right)$ describes the normalized scattering coefficient of the considered target area, $t_{a}$ is azimuth (or slow) time, and $w_{\mathrm{Rx}}\left(t_{a} ; r_{0, \mathrm{Rx}}, x_{0}\right)$ is the normalized receive antenna pattern seen by the target. The SNR for the bistatic image can be obtained by combining (2) and (3)

$$
\begin{aligned}
\operatorname{SNR}\left(x_{0}\right)= & \frac{S\left(x_{0}\right)}{N} \\
\approx & \frac{P_{\mathrm{Tx}} \cdot G_{\mathrm{Tx}} \cdot G_{\mathrm{Rx}} \cdot \lambda^{2} \cdot A_{\mathrm{cell}}}{(4 \pi)^{3} \cdot r_{0, \mathrm{Tx}}^{2} \cdot r_{0, \mathrm{Rx}}^{2} \cdot \mathrm{k} \cdot T_{n} \cdot F_{\mathrm{Rx}} \cdot T_{\mathrm{int}}} \\
& \cdot\left|\int_{T_{\mathrm{int}}} \sqrt{\sigma\left(x_{0}\right)} \cdot w_{\mathrm{Rx}}\left(t_{a} ; r_{0, \mathrm{Rx}}, x_{0}\right) \cdot d t_{a}\right|^{2} .
\end{aligned}
$$

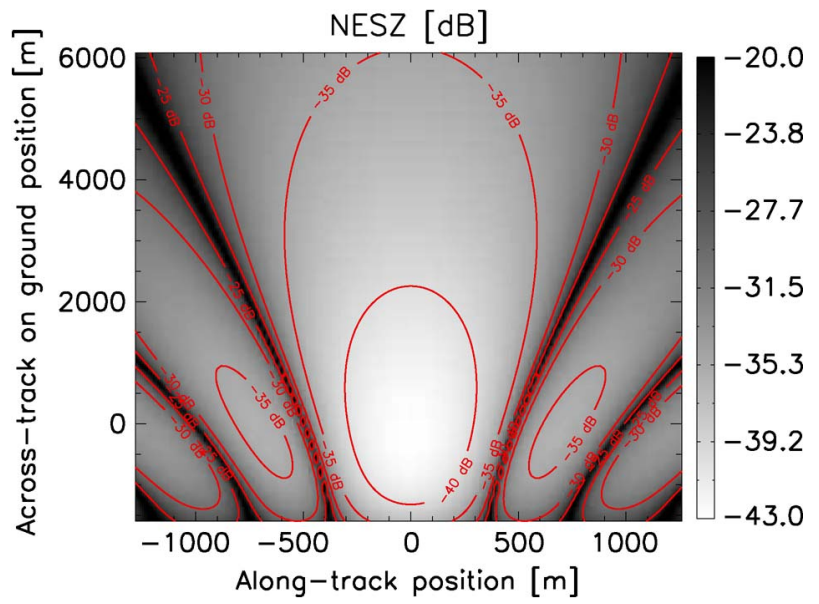

Fig. 4. Expected NESZ map for the bistatic image. Values above $-20 \mathrm{~dB}$ have been clipped to reduce the dynamic range of the plot.

The dependence on $\sigma\left(x_{0}\right)$ accounts for the actual information of the target, which is of little importance in NESZ computations. The NESZ is consequently the inverse of (4) after the removal of factor $\sqrt{\sigma\left(x_{0}\right)}$. Fig. 4 shows the expected NESZ distribution of the processed image. Values above $-20 \mathrm{~dB}$ have been clipped to reduce the dynamic range of the plot. The elevation antenna pattern is directly projected on the ground. If on one side, the SNR is high at the center of the bistatic image; on the other hand, there are regions where almost no signal is present due to the attenuation of the receive antenna pattern. Within the black stripes of the NESZ map, the monostatic TerraSAR-X image has equal or better SNR than the bistatic image. Nevertheless, most of the bistatic image shows better NESZ values than those expected for the monostatic case.

\section{B. Range Resolution}

Slant range resolution can, of course, be described as c/ $B_{r}$. However, and particularly in a bistatic scenario, its meaning is more clearly identified once projected on a flat scene. The onground range resolution is computed using the formula given in [19]

$$
\delta r \approx \frac{\mathrm{c}}{B_{r}} \cdot \frac{1}{\left\|\nabla r_{\mathrm{bi}}\right\|}=\frac{\mathrm{c}}{B_{r}} \cdot \frac{1}{\sin \theta_{\mathrm{Tx}}+\sin \theta_{\mathrm{Rx}}}
$$

where $r_{\mathrm{bi}}$ is the instantaneous bistatic range to the imaged scene history and $\theta_{\mathrm{Tx}}$ and $\theta_{\mathrm{Rx}}$ are the respective transmitter and receiver incident angles. Within the 3-dB elevation range of the F-SAR antenna footprint, the values for the ground range resolution vary between 2.26 and $1.77 \mathrm{~m}$ for near and far ranges, respectively. Within the bistatic footprint, the TerraSAR-X monostatic image has an almost constant ground-range resolution of $1.81 \mathrm{~m}$. The monostatic airborne F-SAR image would have ground range resolutions between $1.73 \mathrm{~m}$ in far and $3 \mathrm{~m}$ in near range.

\section{Along-Track Resolution}

1) Conventional Approach: Assuming there is no significant amplitude modulation in the target response and the 
processing is done mainly on the lower order portion of the bistatic range history, the formula presented in [19] approximates well the along-track resolution

$$
\delta x \approx \frac{1}{T_{\mathrm{int}}} \cdot \frac{1}{\left\|\nabla f_{\mathrm{Dop}}\right\|} \approx \frac{\lambda}{T_{\mathrm{int}}} \cdot\left(\frac{v_{\mathrm{Tx}}}{r_{0, \mathrm{Tx}}}+\frac{v_{\mathrm{Rx}}}{r_{0, \mathrm{Rx}}}\right)^{-1}
$$

where $f_{\text {Dop }}$ is the Doppler frequency history and $r_{0, T x}$ and $r_{0, \mathrm{Rx}}$ are transmitter and receiver monostatic zero-Doppler ranges, respectively. Since spotlight processing has been assumed, $T_{\text {int }}$ remains constant for all targets, and $r_{0, T x}$ and $r_{0, \mathrm{Rx}}$ are constant for a constant range line (assuming a flat scene). Under these assumptions, the along-track resolution is only range dependent. Equation (6) works well for targets located at the center of the scene. The resolution values obtained for an integration time of $2.77 \mathrm{~s}$ are 0.25 and $0.58 \mathrm{~m}$ for near and far ranges, respectively.

2) Extended Approach: The amplitude modulation caused by the bistatic joint antenna pattern for targets placed toward the scene edges has an impact on the maximum achievable along-track resolution, given by (6). To quantify its effect, it is assumed that the considered targets are seen over an angular extent of less than one lobe and outside the 3-dB region of the receive antenna pattern [where (6) does not hold]. These assumptions, valid for the TerraSAR-X/F-SAR bistatic experiment, are needed to approximate the receive antenna pattern by its harmonic part with small error, as explained in the Appendix. Knowing that the data are focused using a nonweighted version of the bistatic backprojection algorithm, the along-track resolution can be approximated as

$$
\delta x_{\mathrm{ext}} \approx\left(\frac{\lambda}{T_{\mathrm{int}}}+\frac{L_{\mathrm{Rx}} \cdot r_{0, \mathrm{Rx}}^{2} \cdot v_{\mathrm{Rx}}}{\sqrt{\left(r_{0, \mathrm{Rx}}^{2}+x_{0}^{2}\right)^{3}}}\right) \cdot\left(\frac{v_{\mathrm{Tx}}}{r_{0, \mathrm{Tx}}}+\frac{v_{\mathrm{Rx}}}{r_{0, \mathrm{Rx}}}\right)^{-1}
$$

where the subscript ext stands for extended and $L_{\mathrm{Rx}}$ is the azimuth length of the Rx antenna. Equation (7) can be expressed as a function of (6) in the following manner:

$$
\delta x_{\mathrm{ext}} \approx \delta x \cdot\left(1+\alpha_{\mathrm{res}}\right)
$$

where $\alpha_{\text {res }}$ represents the resolution degradation factor caused by the receive antenna pattern, i.e.,

$$
\alpha_{\mathrm{res}}=\frac{T_{\mathrm{int}}}{\lambda} \cdot \frac{L_{\mathrm{Rx}} \cdot r_{0, \mathrm{Rx}}^{2} \cdot v_{\mathrm{Rx}}}{\sqrt{\left(r_{0, \mathrm{Rx}}^{2}+x_{0}^{2}\right)^{3}}} .
$$

The analytical derivation of (7)-(9) is presented in the Appendix. Fig. 5 shows the numerically computed along-trackresolution map of the bistatic image. As expected, the alongtrack resolution worsens in across track and along track with respect to the values given by (6), only achieved at the center of the scene. In order to quantify the error in the resolution estimate yielded by (6) for targets placed toward the scene edges, Fig. 6 shows the difference (in percent) between the values of Fig. 5 and those obtained using (6). For simplicity, only the right-hand side of the scene integrated outside the $3 \mathrm{~dB}$

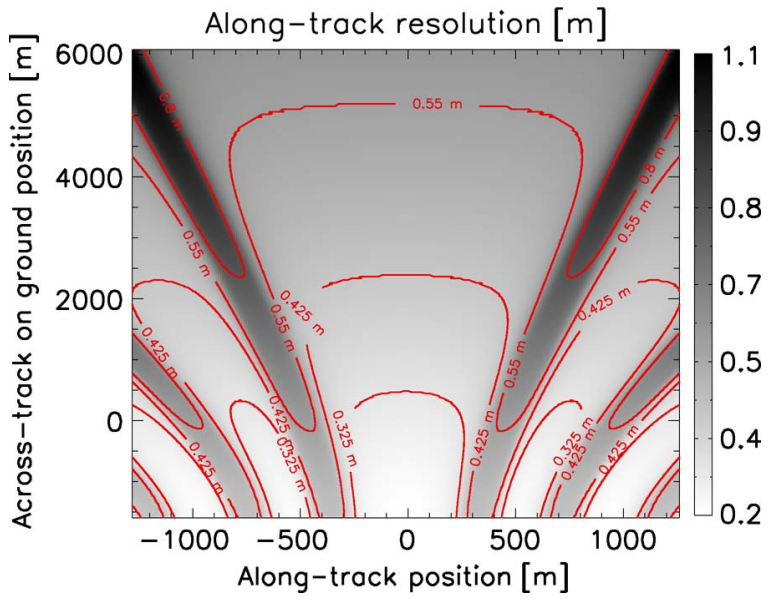

Fig. 5. Numerically computed along-track resolution map for the bistatic image.

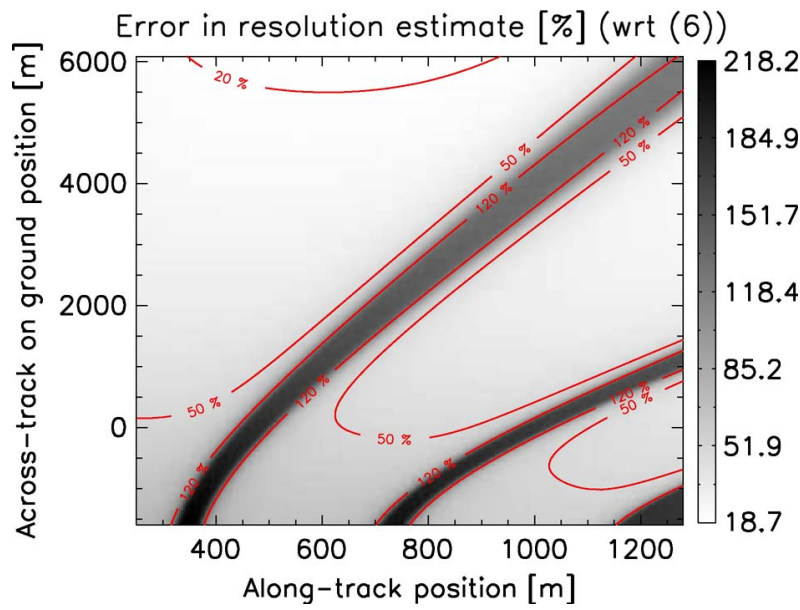

Fig. 6. Error in resolution estimate yielded by (6) for the right-hand part of the scene integrated outside the $3-\mathrm{dB}$ footprint of the receive-antenna pattern.

range of the receive pattern is plotted. The portions of the image with good SNR (cf. Fig. 4) show errors in the estimated resolution below $50 \%$. However, these errors are significantly increased for targets placed near the integrated nulls of the receive antenna, with a maximum value of about $220 \%$. If, on the contrary, we compare Fig. 5 with the estimates yielded by (7) (the difference is shown in Fig. 7), we will notice that the error in the estimated resolution is notably reduced, taking values of less than $5 \%$ for almost the complete scene imaged with good SNR. We conclude that, for the parts of the bistatic image seen with a significant amplitude modulation due to the receive antenna pattern (scene edges), (7) approximates better than (6) the actual along-track resolution of the bistatic image.

\section{Topography Impact on Bistatic Image}

Since target phase histories are a function of transmitter and receiver zero-Doppler ranges, bistatic SAR focusing depends intrinsically on the actual target heights [20]. This effect is shown in Fig. 8, where targets $\mathrm{A}$ and $\mathrm{B}$ are placed at heights $h_{\mathrm{B}}+\Delta h$ and $h_{\mathrm{B}}$, respectively. Note that the separation between the aircraft and the edge of the isorange ellipse is exaggerated to avoid excessive line crossings. Clearly, 


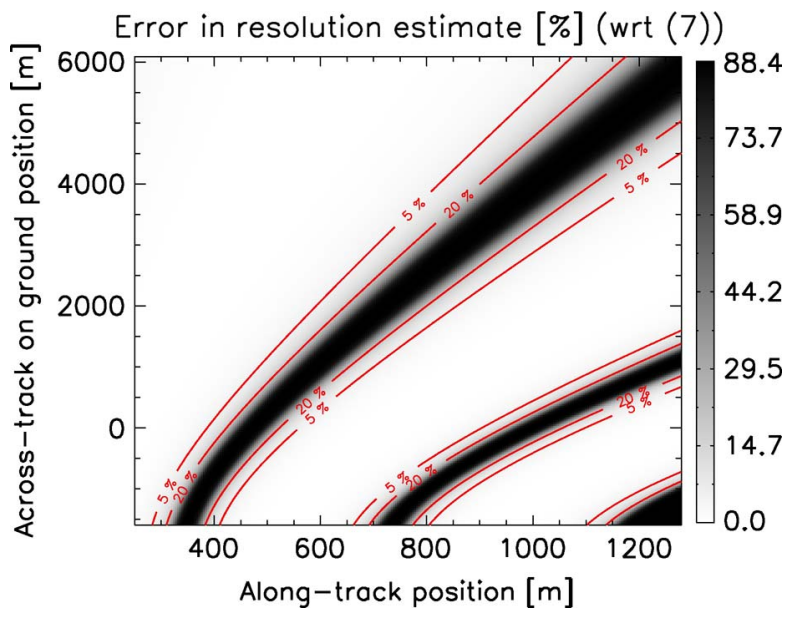

Fig. 7. Error in resolution estimate yielded by (7) for the right-hand part of the scene integrated outside the $3-\mathrm{dB}$ footprint of the receive-antenna pattern.

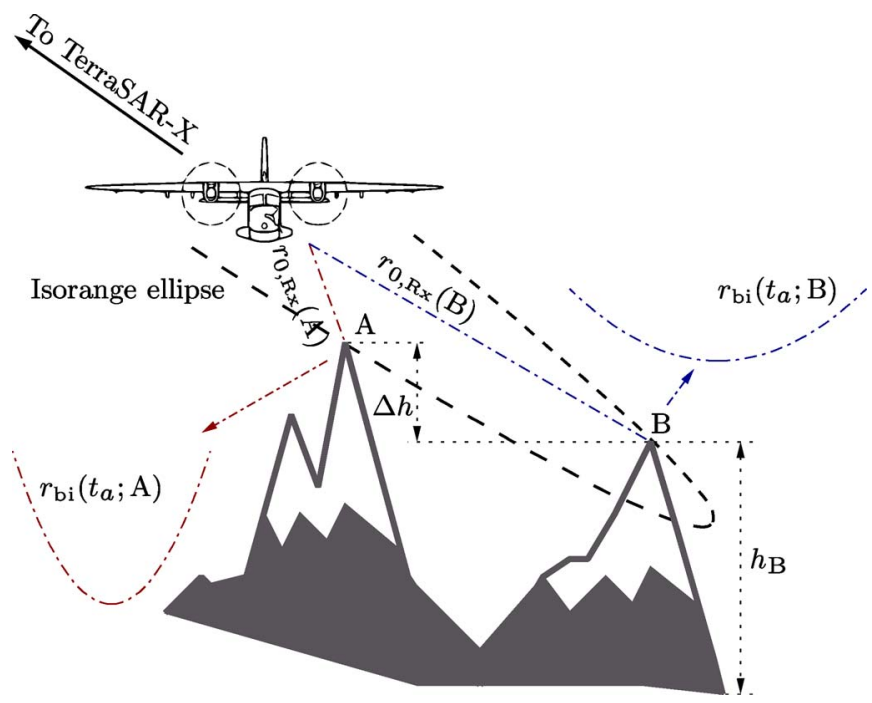

Fig. 8. Effect of topography on target range histories of two different targets, $\mathrm{A}$ and $\mathrm{B}$. Targets $\mathrm{A}$ and $\mathrm{B}$ are placed at different heights and have identical closest bistatic ranges but clearly different range histories.

$r_{0, \mathrm{Rx}}(\mathrm{A})<r_{0, \mathrm{Rx}}(\mathrm{B})$, which necessarily means $r_{0, \mathrm{Tx}}(\mathrm{A})>$ $\mathrm{r}_{0, T \mathrm{~T}}(\mathrm{~B})$, since both targets have the same bistatic range. The difference between the receiver range history curvatures of $\mathrm{A}$ and $\mathrm{B}$ is larger than the difference between the transmitter range history curvatures, which remain almost constant. As a result, targets A and B, sharing the same bistatic range, have different range histories. Hence, processing both targets with a common azimuth reference function induces defocusing. The analytical derivation of the topography impact on the target phase response is cumbersome and exceeds the scope of this paper. However, it is interesting to estimate the topography variations which can be tolerated without introducing a significant defocusing in the final image. Considering Fig. 8, both the height difference $\Delta h$ and the reference height of the target used for focusing $h_{\mathrm{B}}$ can modify the bistatic range history. Fig. 9 shows an example of the tolerable height difference so that the phasehistory error for a near-range target (placed at the azimuth scene center) is kept below $\pi / 2$. The abscissa corresponds to the reference height of the target (used for focusing), and the ordinate

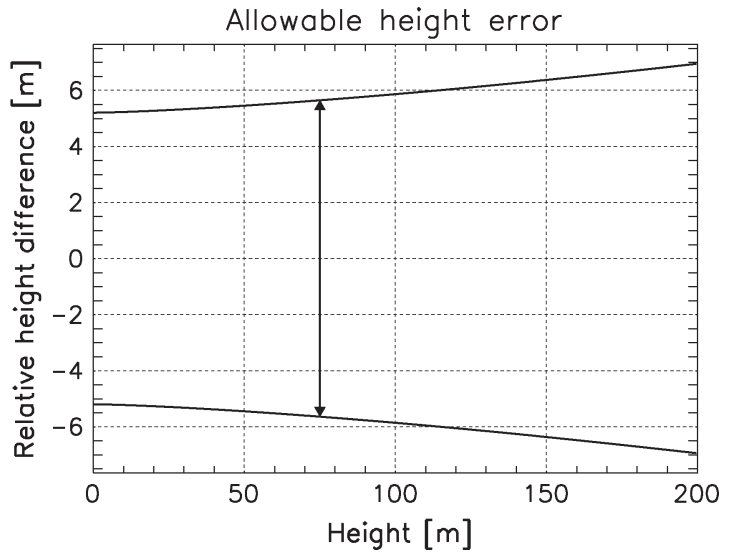

Fig. 9. Allowable topography error for a maximum phase error of $\pi / 2$ in near-range target phase history placed at the azimuth scene center.

shows the tolerable height difference. Within a topography span of $200 \mathrm{~m}$, the allowable height variation is smaller than $\pm 7.5 \mathrm{~m}$. The tolerable height variation increases for increasing ranges, reaching values of $50 \mathrm{~m}$ and $200 \mathrm{~m}$ for mid- and far-range targets, respectively. The Kaufbeuren airfield area is rather flat, with a ground topography variation of about $40 \mathrm{~m}$ (not considering vertical structures like trees or buildings). Therefore, processing the bistatic image without a digital elevation model will not introduce significant defocusing in mid and far range, but care has to be taken in choosing the correct reference height, particularly for accurate focusing in near range.

\section{Data Processing}

Considering the characteristics of the acquisition, with data recorded with two different channels and no dedicated synchronization link between TerraSAR-X and F-SAR clocks, the data processing of this bistatic acquisition must necessarily follow a three-step approach:

1) channel balancing;

2) data synchronization;

3) azimuth-variant bistatic focusing.

Fig. 10 shows a diagram of the different processing blocks, with corresponding plots of data amplitudes on the right-hand side. Channel balancing, the first two top blocks of the diagram, is necessary due to the need of coherently combining the data acquired with two different channels. The second step, data synchronization, is split into two parts: 1) the block "reformatting," where only the nominal TerraSAR-X PRF is used and 2) the "fine synchronization" block after range compression, where the precise frequency offset between the TerraSAR-X and F-SAR clocks is estimated and corrected. After this second block, the bistatic data have reliable range and Doppler references. Once the data are equalized and synchronized, conventional azimuth-variant bistatic SAR focusing is performed.

\section{A. Channel Balancing}

The use of two different channels (each with its own ADC) switched at a higher frequency than the TerraSAR-X PRF 


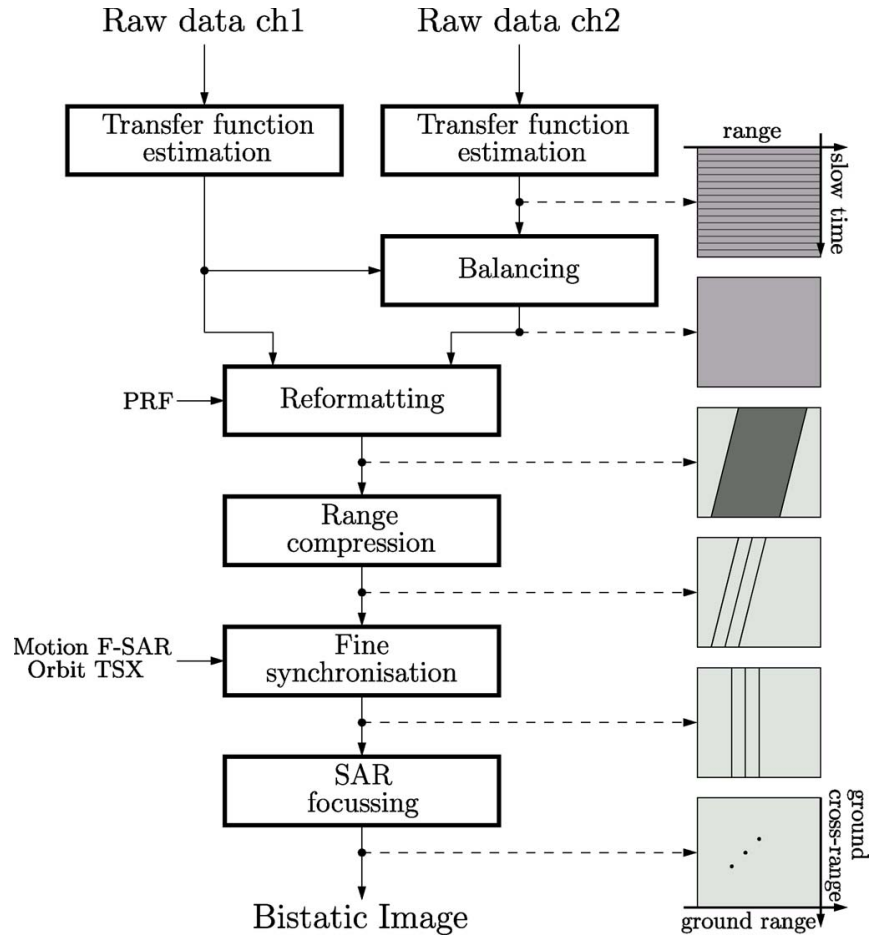

Fig. 10. Block diagram of bistatic processing for TerraSAR-X/F-SAR bistatic acquisition. Illustrative plots of data amplitudes are depicted on the righthand side.
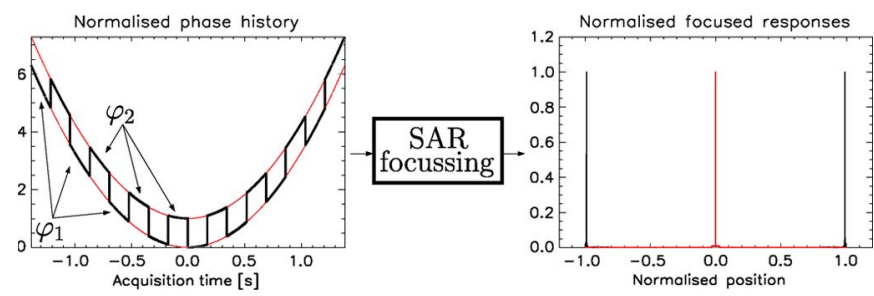

Fig. 11. Effect in (left) phase history and (right) focused response of combining data acquired with two different receiving channels.

induces a quasi pulse-to-pulse modulation on the bistatic data, as shown in Fig. 11. Focusing these data without previous correction yields azimuth ghost targets. The causes of this modulation are the following: 1) different ADCs, 2) different receiver transfer functions, and 3) different electrical path lengths. The first two (pure amplitude effects) can be averaged and corrected using the raw data. The difference in electricalpath lengths causes a constant phase offset between channels 1 and 2. This offset was estimated by computing the signal energy found in adjacent ghosts after correcting with a constant sweeping phase for one of the channels and was found to be $227.5^{\circ}$. A quick-look of the bistatic image was computed to verify the effect of this channel balancing. The results can be seen in Fig. 12. The images show the computed quick-looks for unbalanced (top) and balanced (bottom) data sets. The alongtrack ghosts are clearly visible in the unbalanced version of the quick-look image and significantly attenuated in the balanced image. Note that the use of a second receiver only increased channel unbalance. Sampling the data with two different ADCs would have also produced ghost targets as those shown in Fig. 11, if not previously corrected.

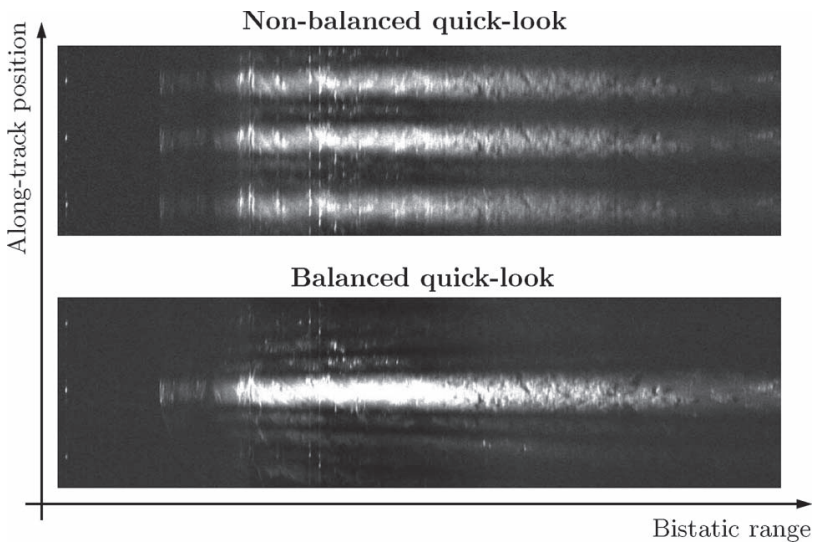

Fig. 12. Quick-looks for (top) nonbalanced and (bottom) balanced data sets. Azimuth ghosts are visible in the nonbalanced quick-looks. These ghosts are significantly reduced in the balanced image. The bright points on the left side (near range) correspond to the direct signal and respective ghosts.

\section{B. Data Synchronization}

Precise bistatic data synchronization, already addressed in [7] and [13], is a necessary step for transforming the acquired bistatic data into quasi-error-free bistatic data. The TerraSAR-X nominal PRF measured by the F-SAR clock is approximated by detecting the first nonzero peak of the autocorrelation function of the radar data. A peak at sample 21113 yields a coarse PRF estimate of $5920.52 \mathrm{~Hz}$. This value is used for reformatting the raw data matrix with 21113 range bins per range line. However, the resulting raw data clearly lack a precise range and Doppler synchronization, which are essential to guarantee highresolution SAR imaging.

Due to the short time of the bistatic acquisition and the high short-term stability of the TerraSAR-X and F-SAR clocks, no fast relative variation of the clocks is expected. Therefore, a constant frequency offset model is assumed. The bistatic data have a constant Doppler shift of $\Delta f_{0}=f_{0, \mathrm{TSX}}-f_{0, \mathrm{~F}-\mathrm{SAR}}$ and an echo-window shift proportional to $\Delta f_{0} / f_{0, T S X},[21]$. The latter causes defocusing, since azimuth-matched filtering (strictly dependent on bistatic range) requires precise knowledge of the range sampling vector. Considering only the lower order portion of the range history, $\Delta f_{0}$ produces a shift of the focused target. For azimuth-variant acquisitions, this azimuth shift in the target position is azimuth dependent. Thus, not correcting the carrier offset causes a nonuniform distortion of the final image. Since both effects are proportional to $\Delta f_{0}$ and can be measured independently, the assumption of a constant offset clock model can be validated. By analyzing the phase of the direct signal and the response of one reference target, a carrier offset of $2841.14 \mathrm{~Hz}$ is estimated within the chosen PRF. Once this frequency offset is corrected in the raw data, the timing of the bistatic data set is finely synchronized using a linear shift so that the phase center of the direct signal coincides with its closest approach. The PRF offset measured after this linear correction is $0.01278715 \mathrm{~Hz}$, which corresponds to $20.842 \mathrm{kHz}$ scaled onto the carrier frequency. After unwrapping the first carrier offset estimate by four times the PRF, the difference with the second estimate reduces to around $1 \mathrm{~Hz}$. This result substantiates the consistency of the bistatic data synchronization procedure. 


\section{Focusing}

Focusing is carried out using a spotlight bistatic backprojection algorithm [22]. Backprojection has the advantage of perfect motion compensation for arbitrary configurations, which is essential for ensuring that no additional phase errors are introduced by the focusing algorithm. A grid laid at a constant height on a spherical Earth is used as output image. No significant phase errors due to topography changes are expected since the whole scene is virtually flat (cf. Section III-D). The spatial sampling is constant and sufficient to avoid aliasing. The synchronized motion data of the satellite and the airplane are available, and no further assumptions on the configuration need be made. For the reference targets, the residual phase error is kept below $\pi / 2$.

\section{RESULTS}

\section{A. Bistatic Image}

Fig. 13 shows the full-bandwidth bistatic spotlight image without any antenna pattern compensation (cf. Fig. 14). The image shows Kaufbeuren city and airfield, surrounded by forested areas and fields. The vertical side corresponds to ground-range, from (top) near to (bottom) far. The effect of the F-SAR azimuth antenna pattern is clearly visible (cf. Section III-A). The three X-band transponders (cf. Section II-B) can be seen below the airfield runway. The interpolated response of the central transponder is shown in Fig. 15. The monostatic (dotted), actual bistatic (solid), and ideal bistatic (dashed) along-track responses are shown. The resolutions are $0.76,0.49$, and $0.36 \mathrm{~m}$, respectively. The loss in resolution and the increase of sidelobe energy are caused by uncompensated residual phase errors in the image. Fig. 16 shows a smoothed version of these residual phase errors (after motion removal) of the direct signal (solid), the third (dotted), and the second transponders (dashed), the three only point-like targets which can be extracted for the whole acquisition time. Residual phase errors have, for this bistatic experiment, two independent sources: 1) residual motion errors of F-SAR and 2) residual differential clock errors. Consequently, the residual phase error of a given target can be expressed as

$$
\Delta \phi_{\text {residual }}\left(t_{a}, i\right]=\frac{2 \pi}{\lambda} \cdot \Delta r_{\text {motion }}\left(t_{a}, i\right]+\Delta \phi_{\text {clock }}\left(t_{a}\right)
$$

where subindex $i$ corresponds to the different independent measures of this residual phase error. Equation (10) is nonlinear, has four unknowns, and neglects the intrinsic noisy character of $\Delta \phi_{\text {residual }}\left(t_{a}, i\right]$ measurement. For the TerraSAR-X/F-SAR bistatic experiment, only three independent residual phase error (cf. Fig. 16) measurements are available, clearly not enough for separating the different error contributions. We can, however, draw some conclusions of the expected errors by considering the values of phase noise and short-term stability of TerraSAR-X and F-SAR clocks (comparable with VECTRON OCXO CO-705 SB19 WL2) and the nominal position accuracy of F-SAR motion unit (around $10 \mathrm{~cm}$ ). Using the results of [21], we conclude that the phase error caused by the clocks is expected to be significantly lower (around factors of two to four) than the error caused by the motion of the airplane, yet not completely negligible.

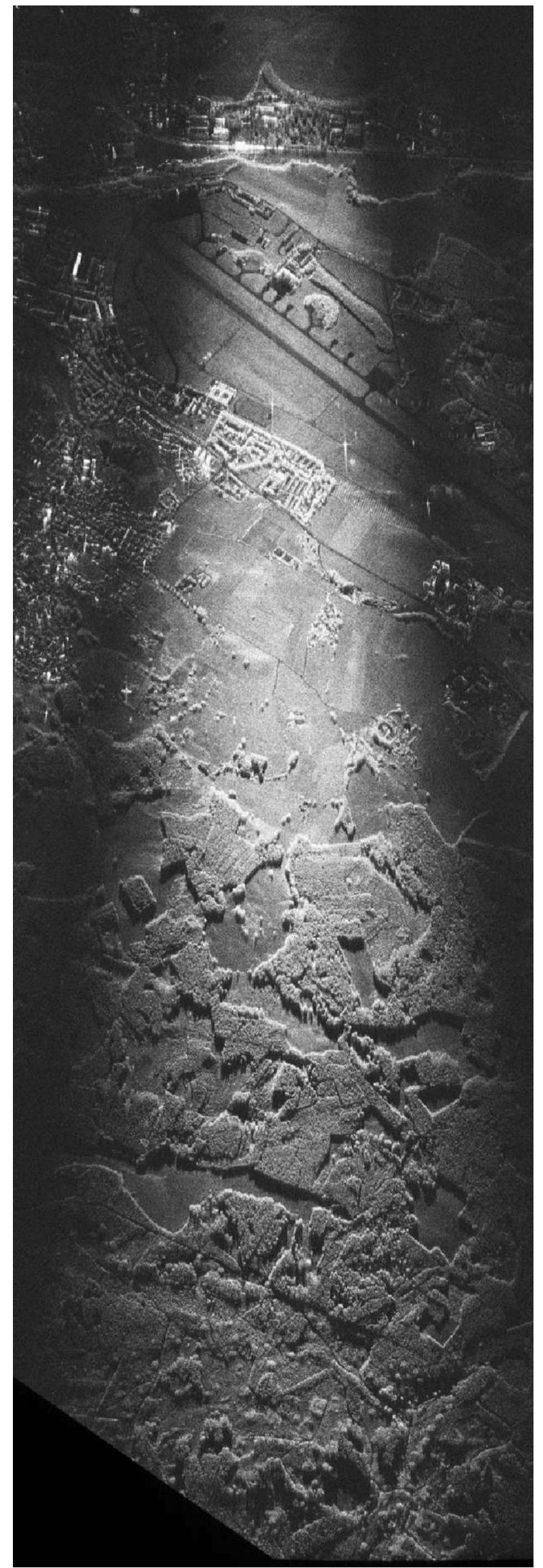

Fig. 13. Bistatic spotlight TerraSAR-X/F-SAR image. $7680 \mathrm{~m}$ in groundrange, $2560 \mathrm{~m}$ in azimuth. Radar illumination from top. 


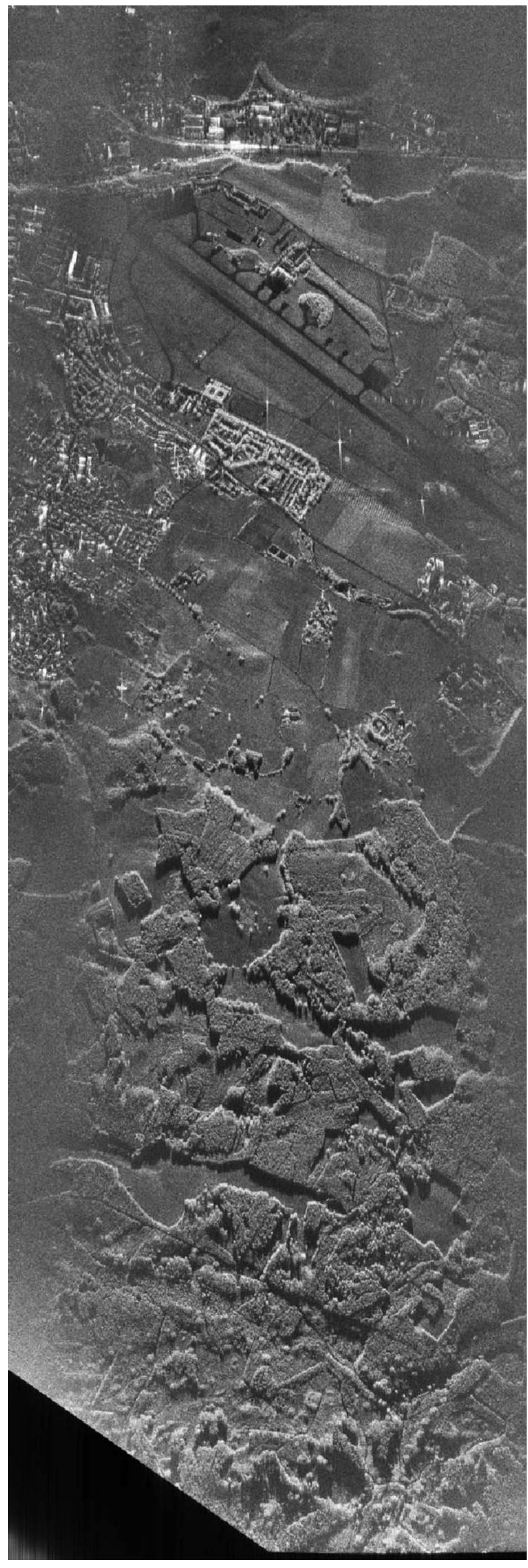

Fig. 14. Bistatic TerraSAR-X/F-SAR image after antenna pattern correction. $7680 \mathrm{~m}$ in ground-range, $2560 \mathrm{~m}$ in azimuth. Radar illumination from top.

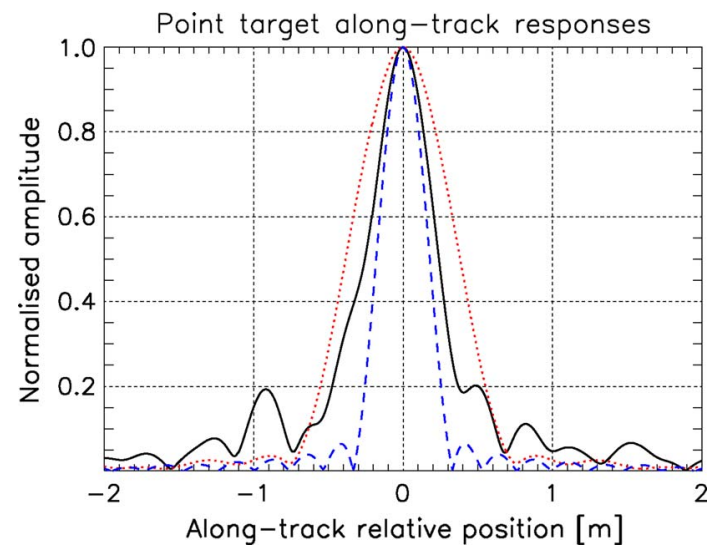

Fig. 15. (Dotted) Monostatic, (solid) bistatic, and (dashed) ideal bistatictransponder responses.

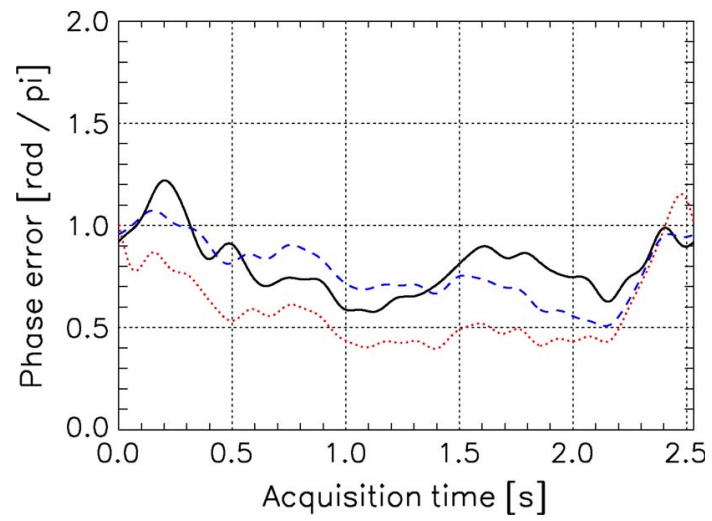

Fig. 16. Phase error measured on different point targets of the bistatic scene. (Solid) Direct signal phase, (dotted) third transponder, and (dashed) second transponder.

\section{B. Pattern Correction}

As previously discussed, the antenna pattern is not directly measurable using the focused image. However, the amplitude modulation induced by the antenna patterns can be compensated using the method presented in Section III-A. Correcting the image with the NESZ map shown in Fig. 4 yields a calibrated bistatic image, which can be seen in Fig. 14 . As expected, the amplitude modulation caused by the bistatic joint pattern disappears (even though the SNR pattern remains unchanged).

\section{Monostatic Versus Bistatic Images}

Fig. 17 shows a part of the previous image with the monostatic TerraSAR-X image (red) laid over the calibrated bistatic TerraSAR-X/F-SAR image (gray). The differences between the images are apparent at first sight. Once again, we would like to recall that TerraSAR-X was not operated in nominal high-resolution spotlight mode: The transmitted bandwidth was reduced from 300 to $100 \mathrm{MHz}$, and the PRF was not optimized for the monostatic acquisition. Range ambiguities arise due to the high PRF and appear as vertical red lines on the monostatic image. The low-SNR sections of the bistatic image can be distinguished by the higher contrast of the red image. This effect is particularly important in near range due to the elevation 

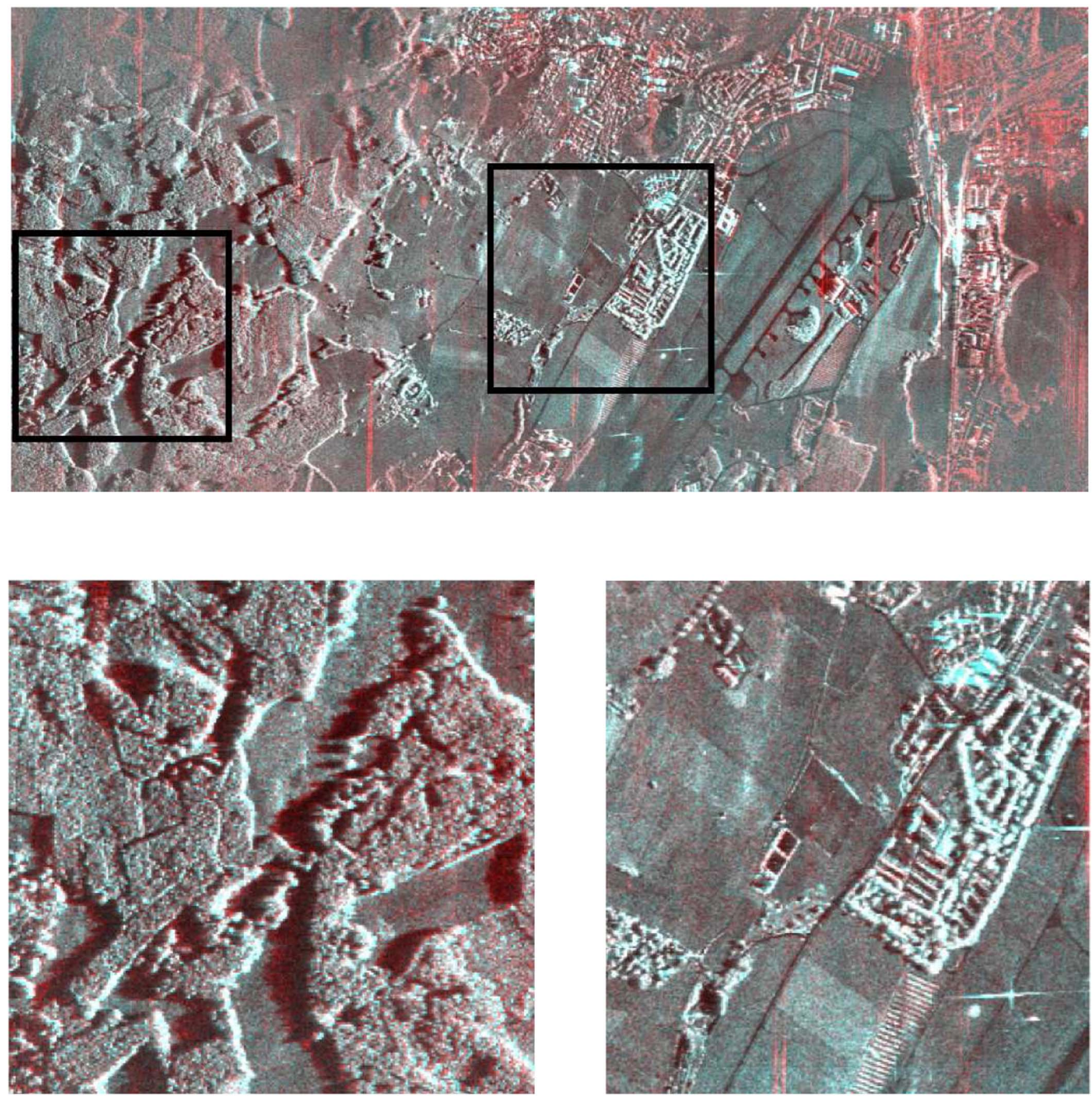

Fig. 17. (Red) Monostatic TerraSAR-X image combined with (gray) the bistatic TerraSAR-X/F-SAR image. Range increases from right to left.

null of the receiver pattern (cf. Section III). In addition to the different along-track resolutions expected in both images (better for most of the bistatic image) and the absence of range ambiguities in the bistatic case, the differences between bistatic and monostatic images can be divided into four classes:

1) signal-to-noise ratio;

2) geometric mapping;

3) scattering mechanisms;

4) Doppler information.

All four effects are noticeable in Fig. 17. The comparison is done using zoomed areas of the monostatic and bistatic images, with the additional help of an optical image of Kaufbeuren.
Fig. 18 shows an enlargement of an area bearing evidence of the SNR difference between both images. The Track and Field facility seen in the bistatic (middle) and optical (right) images is not visible in the monostatic image (left). The two range ambiguities present in the monostatic image (appearing as vertical bright lines) may reduce the dynamic range, but the contrast between the track and the surrounding area appears much more attenuated than in the bistatic counterpart.

The differences in the illumination geometry and scattering mechanisms are a direct consequence of the different incident angles seen by transmitter and receiver. For the presented image, the TerraSAR-X incident angles remain almost constant around $55^{\circ}$, whereas the F-SAR incident angles vary from $15^{\circ}$ 

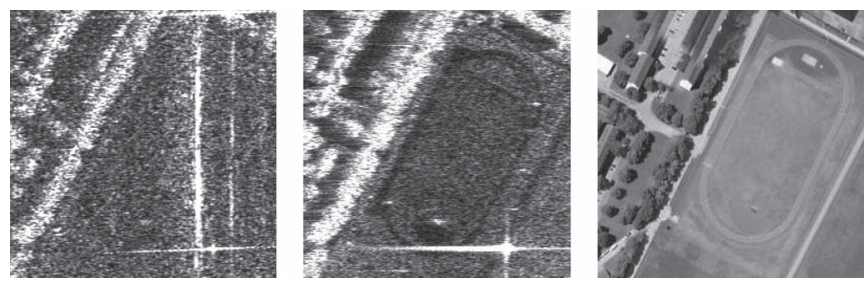

Fig. 18. Track and Field facility next to Kaufbeuren airfield. (Left) Monostatic, (middle) bistatic, and (right) optical images. Note that the monostatic image performance in terms of range resolution and range ambiguity-to-signal ratio does not correspond to the nominal values of TerraSAR-X due to the particularities of the acquisition for the bistatic experiment.
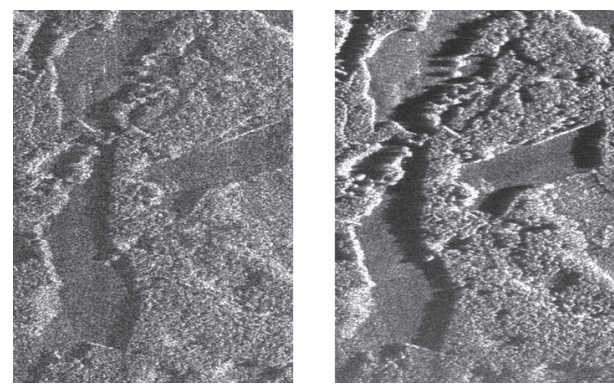

Fig. 19. Forest area near Kaufbeuren: (left) monostatic and (right) bistatic images.
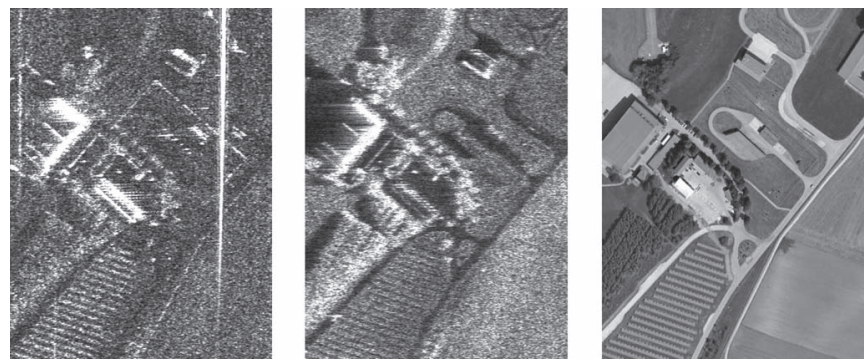

Fig. 20. Buildings and solar panels field: (left) monostatic, (middle) bistatic, and (right) optical images.

to $75^{\circ}$ for near and far range, respectively. One example of the different illumination geometries is shown in Fig. 19. The image shows a forested area near Kaufbeuren in monostatic (left) and bistatic (right) modes. For the left part of both images, the shadows of the trees are clearly larger in the bistatic image. The ratio of the shadow lengths is proportional to the ratio of the tangents of transmitter and receiver incident angles. The receiver incident angle for this part of the image varies between $66^{\circ}$ and $70^{\circ}$. Once again, the difference in radiometric resolution is noticeable. Another interesting example revealing the differences between monostatic and bistatic scattering can be seen in Fig. 20. Monostatic (left), bistatic (middle) and optical (right) images of the same area, some buildings near the airport, are shown. Although the image background is clearly darker in the monostatic image, the buildings appear significantly brighter. A second difference can be noticed between the two main groups of buildings, in what appears to be a metallic fence enclosing two buildings and the access road. This metallic fence, very bright in the monostatic zoom, is absent in the bistatic image. The fence appears bright in the monostatic image probably due to a double-bounce scattering
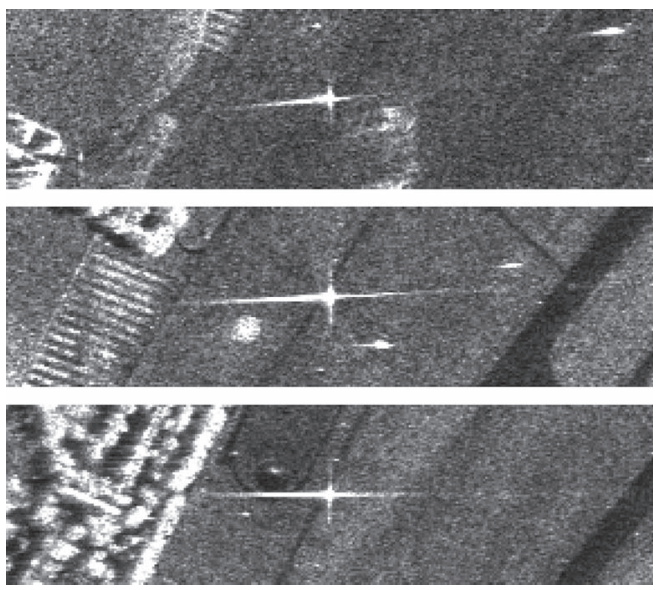

Fig. 21. Transponder responses in bistatic image.

mechanism. Because of the different incident angles in the bistatic case, the double-bounce is invisible to the bistatic radar, and, consequently, the fence disappears in the bistatic image. The third interesting point can be seen at the bottom of the images, featuring a solar-panel field. Very bright in the monostatic image due to the orientation of the panels, the area appears much darker in the bistatic image.

The last example shown in Fig. 21 helps in illustrating the differences in Doppler components of both images. Due to the azimuth-variance of this bistatic configuration, the view angles of the targets change with their azimuth position. This means that the frequency content of the targets also changes with azimuth position. The three images show the three different transponders in the bistatic image. Due to the different spectral information of each transponder and due to the different equivalent squint angles, the sidelobes in the range and azimuth of the first (top) and second (middle) transponders are no longer orthogonal.

\section{SUMMARY}

This paper has given a comprehensive report of the TerraSAR-X/F-SAR bistatic SAR experiment including description, performance estimation, data processing, and results. The experiment was the first X-band bistatic spaceborneairborne acquisition and the first including full synchronization (performed in processing steps) and high-resolution imaging. The proposed processing method portrays a general approach for any kind of nonsynchronized bistatic SAR system with clocks having a stable relative behavior (e.g., short acquisition times or systems with dedicated synchronization links). The performance analysis presented for this azimuth-variant acquisition has been validated, including the quantitative analysis of the effects of antenna patterns on the along-track resolution and SNR. Finally, a comparison of the monostatic TerraSAR-X and bistatic TerraSAR-X/F-SAR images has shown some interesting properties. The bistatic image has an advantage in terms of resolution and SNR and in the complete absence of range ambiguities (highly dependent on configuration and acquisition). On the other hand, the monostatic image shows a more homogeneous behavior in terms of SNR. These effects, as well as the described differences in 
scattering mechanisms and information content of the images, demonstrate the capabilities of bistatic SAR to be a valuable complement to existing monostatic systems.

\section{APPENDIX}

The steps leading to (7) are described and discussed in this Appendix. The assumptions and values used in Section III remain valid for the following derivation. The perfectly synchronized bistatic baseband SAR response of one target placed at azimuth position $x_{0}$ can be written as

$$
s_{\text {raw }}\left(t_{a} ; x_{0}\right)=g_{\mathrm{bi}} \cdot w_{\mathrm{bi}}\left(t_{a} ; x_{0}\right) \cdot \exp \left[-\mathrm{j} \cdot \frac{2 \pi}{\lambda} \cdot r_{\mathrm{bi}}\left(t_{a} ; x_{0}\right)\right]
$$

where $w_{\text {bi }}$ models the bistatic joint pattern as a product of the normalized transmitter and receiver contributions, $w_{\mathrm{bi}}=w_{\mathrm{Tx}}$. $w_{\mathrm{Rx}} ; g_{\mathrm{bi}}$ is a calibration constant proportional to the product of Tx and Rx antenna gains and $r_{\mathrm{bi}}\left(t_{a} ; x_{0}\right)$ denotes the bistatic range history of this target. After SAR processing, the focused response of the same target can be approximated by

$$
s_{\mathrm{SAR}}(x) \approx g_{\mathrm{bi}} \cdot \exp \left[\mathrm{j} \cdot \Phi\left(x, x_{0}\right)\right] \cdot W_{\mathrm{bi}}\left(\frac{x-x_{0}}{K_{\mathrm{bi}}}\right)
$$

with

$$
K_{\mathrm{bi}}=\lambda \cdot\left(\frac{v_{\mathrm{Tx}}}{r_{0, \mathrm{Tx}}}+\frac{v_{\mathrm{Rx}}}{r_{0, \mathrm{Rx}}}\right)^{-1}
$$

where $W_{\mathrm{bi}}$ is the Fourier transform of the bistatic joint pattern. In addition, $t_{a}$ is transformed into $x$ (along-track coordinate) using the scaling factor $K_{\mathrm{bi}}$, a result consistent with (6). Equation (12) originates from a quadratic expansion of the bistatic range history and is valid for the following results. Assuming a sinc-form receive antenna pattern, the bistatic joint pattern can be approximated by

$$
w_{\mathrm{bi}}\left(t_{a}\right) \approx \operatorname{sinc}\left[\frac{L_{\mathrm{Rx}} \cdot\left(v_{\mathrm{Rx}} \cdot t_{a}-x_{0}\right)}{\lambda \cdot \sqrt{r_{0, \mathrm{Rx}}^{2}+\left(v_{\mathrm{Rx}} \cdot t_{a}-x_{0}\right)^{2}}}\right] \cdot w_{\mathrm{Tx}}\left(t_{a}\right)
$$

where $L_{\mathrm{Rx}}$ is the azimuth length of the $\mathrm{Rx}$ antenna and $w_{\text {Tx }}$ is a smoothly varying function within the interval $\left[-T_{\text {int }} / 2, T_{\text {int }} / 2\right]$. The contribution of the sinc function to the joint pattern can be separated into: 1) a harmonic part including the sinusoidal term of the receiver pattern and 2) a smooth modulation proportional to $w_{\mathrm{Tx}} / t_{a}$. This second factor is responsible for small changes in the ideal (sinc-form-like) impulse response. The harmonic factor $\sin \left[L_{\mathrm{Rx}} \cdot\left(v_{\mathrm{Rx}} \cdot t_{a}-\right.\right.$ $\left.\left.x_{0}\right) /\left(\lambda \cdot \sqrt{r_{0, \mathrm{Rx}}^{2}+\left(v_{\mathrm{Rx}} \cdot t_{a}-x_{0}\right)^{2}}\right)\right]$ is responsible for the degradation of the impulse response. Linearizing the argument of this harmonic function, the resulting focused response can be approximated by

$$
\begin{aligned}
& s_{\mathrm{SAR}}(x) \approx h\left(x-\Delta x_{\mathrm{ant}}\right) \cdot \exp \left[\mathrm{j} \cdot \phi_{\mathrm{ant}}\right] \\
&-h\left(x+\Delta x_{\mathrm{ant}}\right) \cdot \exp \left[-\mathrm{j} \cdot \phi_{\mathrm{ant}}\right]
\end{aligned}
$$

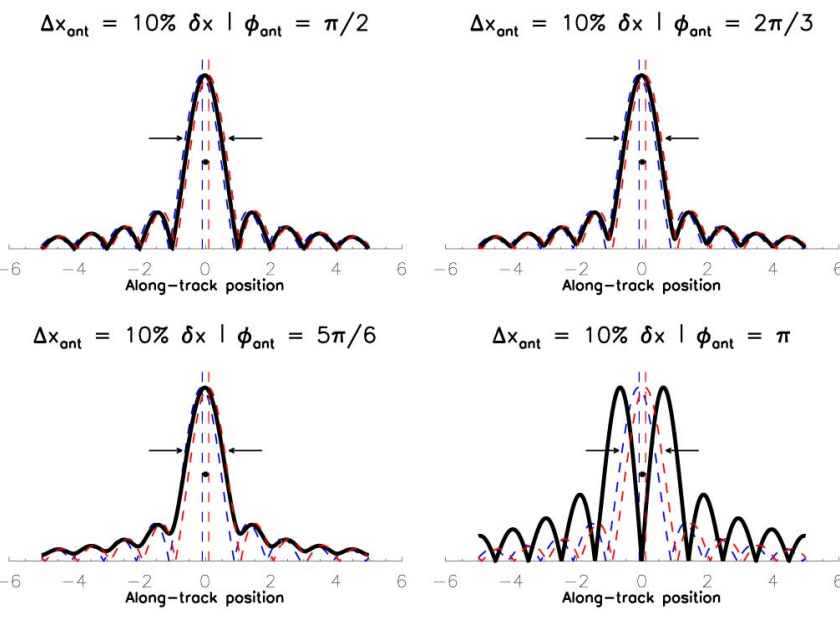

Fig. 22. Along-track impulse responses with increasing values of $\phi_{\text {ant }}$ from (top left) $\pi / 2$ to (bottom right) $\pi$ and a constant value of $\Delta x$ ant $10 \%$ of $\delta x$. The resulting impulse response is drawn in solid. The dashed lines show the shifted nonmodulated impulse responses whose complex addition approximates the focused signal.
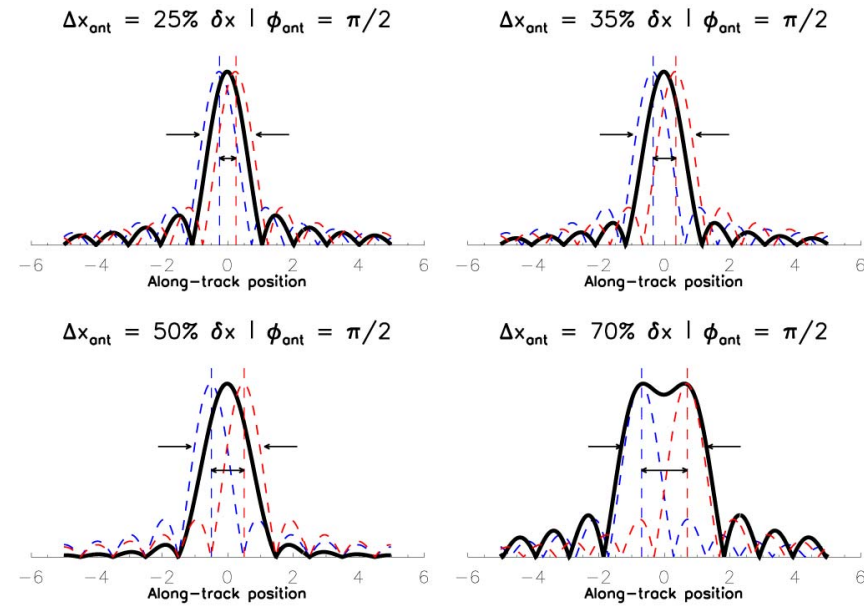

Fig. 23. Along-track impulse responses with decreasing values of $\Delta x_{\text {ant }}$ from (top left) $25 \%$ to (bottom right) $70 \%$ of $\delta x$ and a constant value of $\phi_{\text {ant }}$ of $\pi / 2$. Impulse responses are computed like in Fig. 22.

where $h(x)$ is the Fourier transform of $w_{\mathrm{Tx}} / t_{a}$ and constant $0.5 \cdot \mathrm{j}$ has been omitted for compactness. $\Delta x_{\mathrm{ant}}$ and $\phi_{\mathrm{ant}}$ are given by

$$
\begin{aligned}
\Delta x_{\mathrm{ant}} & \approx \frac{L_{\mathrm{Rx}} \cdot r_{0, \mathrm{Rx}}^{2} \cdot v_{\mathrm{Rx}}}{2 \cdot\left(r_{0, \mathrm{Rx}}^{2}+x_{0}^{2}\right)^{1.5}} \cdot\left(\frac{v_{\mathrm{Tx}}}{r_{0, \mathrm{Tx}}}+\frac{v_{\mathrm{Rx}}}{r_{0, \mathrm{Rx}}}\right)^{-1} \\
\phi_{\mathrm{ant}} & \approx-\pi \cdot \frac{L_{\mathrm{Rx}} \cdot x_{0}}{\lambda \cdot \sqrt{r_{0, \mathrm{Rx}}^{2}+x_{0}^{2}}} .
\end{aligned}
$$

The value of $\phi_{\text {ant }}$ mainly depends on the portion of the receiver antenna pattern seen by the target. Values around $\pi / 2+k \cdot \pi$ (with $k$ an integer) correspond to sidelobe maxima, and values around $k \cdot \pi$ correspond to the nulls of the receiver antenna pattern (except $\phi_{\text {ant }}=0$, which corresponds to an unsquinted acquisition). For the experiment, $\Delta x_{\text {ant }}$ takes values varying from $35 \%$ to $25 \%$ of the conventional along-track resolution $\delta x$ for near-range targets and an almost constant $10 \%$ of $\delta x$ for far-range targets. 


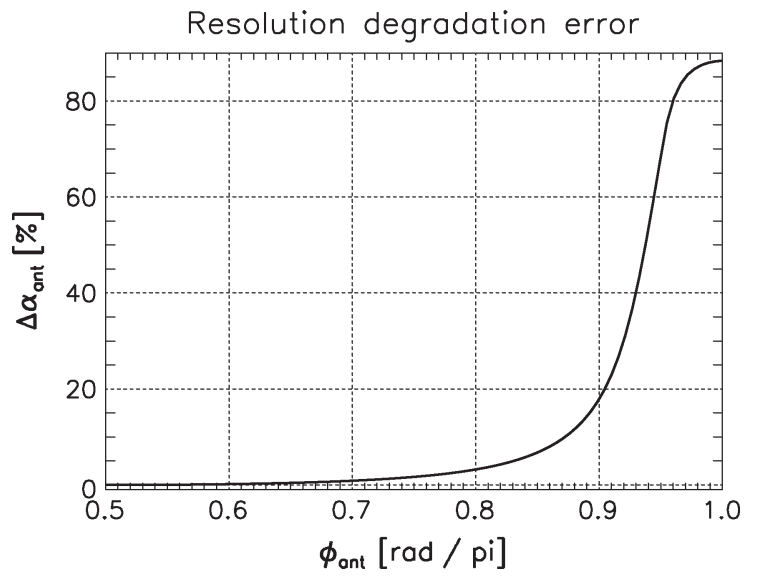

Fig. 24. Error in along-track resolution-degradation factor, $\Delta \alpha_{\text {ant }}$, caused by approximation (17).

To illustrate the effect of varying the value of $\phi_{\text {ant }}$ on the focused impulse response, Fig. 22 shows the results for four different values of this equivalent phase: $\pi / 2$ (top left), $2 \pi / 3$ (top right), $5 \pi / 6$ (bottom left), and $\pi$ (bottom right) and a constant value of $\Delta x_{\text {ant }}$ of $10 \%$ of the conventional $\delta x$, which is normalized to unity. In general, the form of the impulse response improves if $\phi_{\text {ant }}$ approaches $\pi / 2$ (coinciding with the maxima of the sidelobes), going from the sinc-like form in topleft of the plot to the two-peak impulse response plotted in the bottom-right. The target resolution clearly worsens from topleft to bottom-right. Fig. 23 shows a similar plot for a constant value of $\phi_{\text {ant }}=\pi / 2$ (sidelobe maximum) and values of $\Delta x_{\text {ant }}$ of $25 \%, 35 \%, 50 \%$, and $70 \%$ of $\delta x$. The latter two values are higher than those expected in the bistatic spaceborne-airborne experiment. They are, however, reported in the following for illustration purposes. Increasing the values of $\Delta x_{\text {ant }}$ worsen the resolution and the form of the impulse response. Note that a two-peak impulse response is also obtained for the case of $\Delta x_{\text {ant }}=0.7 \cdot \delta x$, although no significant signal energy is lost in this case. We can assume that, except for the cases where the target is seen through a null of the receive antenna (low SNR), the degradation in resolution is mainly caused by $\Delta x_{\text {ant }}$. Using (14), the relationship between (6) and (7) becomes clear

$$
\delta x_{\mathrm{ext}} \approx \delta x+2 \cdot \Delta x_{\mathrm{ant}} .
$$

Within the bounds of the approximation, the error introduced by (17) in the estimation of the degradation factor $\alpha_{\text {res }}$ as a function of $\phi_{\text {ant }}$ is shown in Fig. 24. This error is defined as the difference between the numerically computed degradation and $\alpha_{\text {res }}$ obtained using (9) expressed in present. For $80 \%$ of the targets (seen in the plot with values of $\phi_{\text {ant }}$ between $\pi / 2$ and $0.9 \cdot \pi$ ), the error of the analytically computed degradation factor is smaller than $20 \%$ and, in the worst case, smaller than $90 \%$ (coinciding with focused targets characterized by low SNR, cf. Fig. 7).

\section{ACKNOWLEDGMENT}

The authors would like to thank the many colleagues in TerraSAR-X ground segment team and in F-SAR operational team for their kind help and support, namely, U. Steinbrecher, R. Metzig, M. Younis, C. Ortega-Míguez, N. Tous-Ramón, A. Meta, M. Schwerdt, M. Limbach, S. Pasch, T. Nottensteiner, R. Horn, P. Prats, R. Scheiber, C. Andres, and J. Fischer, to F. Kurz from the Institute of Remote Sensing Technology of DLR for providing the optical image of Kaufbeuren area, to P. Dheenathayalan and M. Galletti for reviewing a version of this paper, and to the anonymous reviewers for their detailed and helpful comments, which significantly improved the quality of this paper.

\section{REFERENCES}

[1] J. L. Auterman, "Phase stability requirements for bistatic SAR," in Proc. IEEE Nat. Radar Conf., Atlanta, GA, 1984, pp. 48-52.

[2] D. Martinsek and R. Goldstein, "Bistatic radar experiment," in Proc. EUSAR, Berlin, Germany, 1998.

[3] N. Willis, Bistatic Radar. Norwood, MA: Artech House, 1991, pp. $46-49$.

[4] M. Wendler, G. Krieger, R. Horn, B. Gabler, and P. Dubois-Fernandez, "Results of a bistatic airborne SAR experiment," in Proc. IRS, Dresden, Germany, 2003, pp. 247-253.

[5] J. Ender, I. Walterschied, and A. Brenner, "New aspects of bistatic SAR: Processing and experiments," in Proc. IGARSS, Anchorage, AK, 2004, pp. $1758-1762$.

[6] G. Yates, A. M. Horne, A. P. Blake, R. Middleton, and D. B. Andre, "Bistatic SAR image formation," in Proc. EUSAR. Ulm, Germany, 2004, pp. $581-584$.

[7] H. Cantalloube, M. Wendler, V. Giroux, P. Dubois-Fernández, and G. Krieger, "Challenges in SAR processing for airborne bistatic acquisitions," in Proc. EUSAR, Ulm, Germany, 2004, pp. 577-580.

[8] H. Cantalloube, P. Dubois-Fernandez, V. Giroux, and G. Krieger, "Bistatic moving target indication using across-track and along-track interferometry," in Proc. EUSAR, Dresden, Germany, 2006.

[9] P. Dubois-Fernandez, H. Cantalloube, B. Vaizan, G. Krieger, R. Horn, M. Wendler, and V. Giroux, "ONERA-DLR bistatic SAR campaign: Planning, data acquisition, and first analysis of bistatic scattering behavior of natural and urban targets," Proc. Inst. Elect. Eng.-Radar, Sonar Navig., vol. 153, no. 3, pp. 214-223, Jun. 2006.

[10] M. Cherniakov, P. Dubois-Fernandez, H. Cantalloube, B. Vaizan, G. Krieger, and A. Moreira, Bistatic Radar: Emerging Technology. New York: Wiley, 2008, pp. 159-213.

[11] G. Krieger, A. Moreira, H. Fiedler, I. Hajnsek, M. Werner, M. Younis, and M. Zink, "TanDEM-X: A satellite formation for high-resolution SAR interferometry," IEEE Trans. Geosci. Remote Sens., vol. 45, no. 11, pp. 3317-3341, Nov. 2007.

[12] J. Sanz-Marcos, P. Lopez-Dekker, J. J. Mallorqui, A. Aguasca, and P. Prats, "SABRINA: A SAR bistatic receiver for interferometric applications," IEEE Geosci. Remote Sens. Lett., vol. 4, no. 2, pp. 307-311, Apr. 2007.

[13] P. Lopez-Dekker, J. J. Mallorqui, P. Serra-Morales, and J. Sanz-Marcos, "Phase synchronization and Doppler centroid estimation in fixed receiver bistatic SAR systems," IEEE Trans. Geosci. Remote Sens., vol. 46, no. 11, pp. 3459-3471, Nov. 2008.

[14] A. Goh, M. Preiss, N. Stacy, and D. Gray, "Bistatic SAR experiment with the Ingara imaging radar: Preliminary results," in Proc. EUSAR, Friedrichshafen, Germany, 2008.

[15] L. Ulander, B. Flood, P. Frölind, T. Jonsson, A. Gustavsson, J. Rasmusson, G. Stenström, A. Barmettler, and E. Meier, "Bistatic experiment with ultra-wideband VHF synthetic aperture radar," in Proc. EUSAR, Friedrichshafen, Germany, 2008.

[16] S. V. Baumgartner, M. Rodriguez-Cassola, A. Nottensteiner, R. Horn, M. Schwerdt, R. Scheiber, U. Steinbrecher, R. Metzig, M. Limbach, J. Mittermayer, G. Krieger, and A. Moreira, "Bistatic experiment using TerraSAR-X and DLR's new F-SAR system," in Proc. EUSAR, Friedrichshafen, Germany, 2008.

[17] M. Rodriguez-Cassola, S. V. Baumgartner, A. Nottensteiner, R. Horn, M. Schwerdt, P. Prats, J. Fischer, U. Steinbrecher, R. Metzig, M. Limbach, G. Krieger, and A. Moreira, "Bistatic spaceborne-airborne experiment TerraSAR-X/F-SAR: Data processing and results," in Proc. IGARSS, Boston, MA, 2008, pp. III-451-III-454.

[18] R. Horn, A. Nottensteiner, and R. Scheiber, "F-SAR-DLR's advanced airborne SAR system onboard DO228," in Proc. EUSAR, Friedrichshafen, Germany, 2008. 
[19] G. Krieger, H. Fiedler, D. Hounam, and A. Moreira, "Analysis of system concepts for bi- and multi-static SAR missions," in Proc. IGARSS, Toulouse, France, 2003, pp. 770-772.

[20] M. Rodriguez-Cassola, G. Krieger, and M. Wendler, "Azimuth-invariant, bistatic airborne SAR processing strategies based on monostatic algorithms," in Proc. IGARSS, Seoul, Korea, 2005, pp. 1047-1050.

[21] G. Krieger and M. Younis, "Impact of oscillator noise in bistatic and multistatic SAR," IEEE Geosci. Remote Sens. Lett., vol. 3, no. 3, pp. 424 428, Jul. 2006.

[22] J. L. Bauck and W. K. Jenkins, "Convolution-backprojection image reconstruction for bistatic synthetic aperture radar," in Proc. ISCAS, Portland, OR, 1989, pp. 1512-1515.

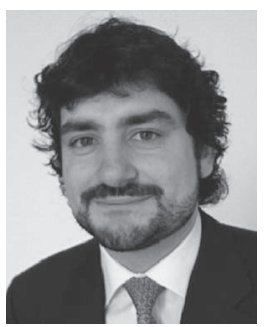

Marc Rodriguez-Cassola was born in Barcelona, Spain, in 1977. He received the Ingeniero degree in telecommunication engineering from Universidad Pública de Navarra, Pamplona, Spain, in 2000.

From 2000 to 2001, he was a Radar Hardware Engineer with CETP/CNRS, Saint Maur des Fossés, France. From 2001 to 2003, he worked as a Software Engineer for Altran Consulting, Germany. Since 2003, he has been with the Microwaves and Radar Institute, German Aerospace Center (DLR), Oberpfaffenhofen, Germany, where he has been working on airborne and spaceborne bistatic SAR system analysis and data processing. His current research interests include radar signal processing, innovative high-precision SAR imaging algorithms, and bistatic radar system analysis and applications.

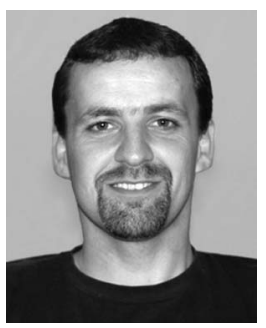

Stefan V. Baumgartner received the Dipl--Ing. (M.S.) degree in electrical engineering and communication technology from Graz University of Technology, Graz, Austria, in 2004.

Since 2004, he has been with the Microwaves and Radar Institute (HR), German Aerospace Center (DLR), Oberpfaffenhofen, Germany. He is currently with the Radar Concepts Department, where his field of activity is the development of ground moving target indication and parameter estimation algorithms for future road-traffic-monitoring applications using multichannel air- and spaceborne synthetic aperture radars (SAR). His research interests include SAR along-track interferometry, time-frequency analysis, and other advanced signal and imaging processing techniques.

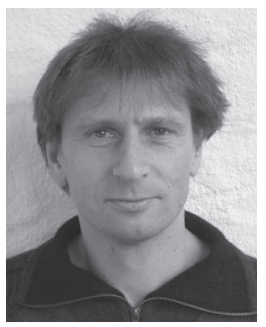

Gerhard Krieger (M'03) received the Dipl.-Ing. (M.S.) and Dr.-Ing. (Ph.D.) degrees (with honors) in electrical and communication engineering from the Technical University of Munich, Munich, Germany, in 1992 and 1999, respectively.

From 1992 to 1999 , he was with the LudwigMaximilians University, Munich, where he conducted multidisciplinary research on neuronal modeling and nonlinear information processing in biological and technical vision systems. Since 1999, he has been with the Microwaves and Radar Institute, German Aerospace Center (DLR), Oberpfaffenhofen, Germany, where he developed signal- and image-processing algorithms for a novel forward-looking radar system employing the principle of digital beamforming on receive. From 2001 to 2007, he led the New SAR Missions Group which pioneered the development of advanced bistatic and multistatic radar systems as exemplified by the forthcoming TanDEM-X mission as well as innovative multichannel SAR techniques and algorithms for high-resolution wide-swath SAR imaging. Since 2008, he has been the Head of the new Radar Concepts. His current research interests focus on the development of multichannel radar techniques and algorithms for innovative MIMO SAR systems, the demonstration of novel interferometric and tomographic Earth-observation applications, and the conceptual design of advanced bi- and multistatic radar missions.

Dr. Krieger is the recipient of several national and international awards, including the IEEE Transactions Prize Paper Award of the Geoscience and Remote Sensing Society and the Otto Lilienthal Sabbatical of the German Aerospace Center, DLR.

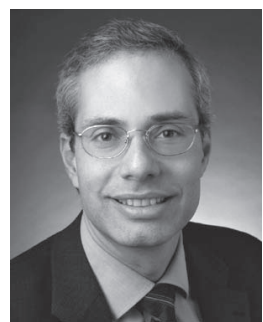

Alberto Moreira (M'92-SM'96-F'04) was born in São José dos Campos, Brazil, in 1962. He received the B.S.E.E. and M.S.E.E. degrees from the Aeronautical Technological Institute ITA, Brazil, in 1984 and 1986, respectively, and the Eng.Dr. degree (Honors) from the Technical University of Munich, Munich, Germany, 1993.

From 1996 to 2001, he was the Chief Scientist and Engineer with the SAR Technology Department, Microwaves and Radar Institute, German Aerospace Center (DLR), Oberpfaffenhofen, Germany, where since 2001, he has been the Director of the Microwaves and Radar Institute. The Institute contributes to several scientific programs and space projects for actual and future air- and spaceborne SAR missions like TerraSAR-X and Sentinel-1. In 2003, he was a Full Professor with the University of Karlsruhe, Karlsruhe, Germany, in the field of Microwave Remote Sensing. In 2006, the mission proposal TanDEM-X lead by his Institute has been approved for the realization phase. He is the Principal Investigator for this mission. Under his leadership, the DLR airborne SAR system, E-SAR, has been upgraded to operate in innovative imaging modes like polarimetric SAR interferometry and SAR tomography. His professional interests and research areas encompass radar end-to-end system design and analysis, innovative microwave techniques and system concepts, signal processing, and remote-sensing applications. He has more than 250 publications in international conferences and journals and is the holder of 15 patents in the radar and antenna field.

Prof. Moreira is serving as a member of the IEEE Geoscience and Remote Sensing Society (GRS-S) Administrative Committee (1999-2001, 2004-2007, 2008-2009 as Executive Vice-President), was the founder and chair of the German Chapter of the GRS-S (2003-2008), was an Associate Editor for the IEEE Geoscience And Remote Sensing Letters (2003-2007) and is serving since 2005 as Associate and Guest Editor for the IEEE Transactions on Geoscience And Remote Sensing. In 1995, he was the recipient of the DLR Science Award. He and his colleagues received the GRS-S Transactions Prize Paper Awards in 1997, 2001, and 2007. He is also the recipient of the IEEE Nathanson Award for the Young Radar Engineer of the Year (1999) and the IEEE Kiyo Tomiyasu Field Award (2007). From 2003 until 2008, he served as a member of the Board of Directors of the Information Technology Society of the German Association for Electrical, Electronic and Information Technologies. He has participated in 19 IGARSS conferences. He has contributed to the successful series of the European SAR conferences (EUSAR) since 1996 as member of the Technical Program Committee, Technical Chairman (2000), Awards Chairman (2002-2004), General Chairman (2006), and Co-Chairman (2008). 